\title{
Service Innovation: Taking Stock of Existing Literature
}

\author{
Md Rokonuzzaman and Audhesh Paswan
}

\begin{abstract}
Most agree that innovation is crucial for enhancing competitive advantage. Further, Service-Dominant Logic (SDL) literature highlights the role of service in enhancing competitive advantage through value co-creation. Finally, most agree that services for a major part of most developed economies. Taken together, the topic of service innovation is critical, especially given the increasing prominence of service in most developed economies. While a lot of work has been done in this area, the literature on service innovation is somewhat scattered. This study attempts to integrate the existing literature and proposes a conceptual strategic framework highlighting the relationships between different domains of service innovation. In doing so, we also identify the gaps in the literature and suggest possible avenues for research. The proposed framework consists of three categories of antecedents that have been examined in the literature-internal to the firm, external to the firm, and interface dimensions. In addition, researchers have examined the outcomes of service innovation - the effect of service innovation on the process or the operations of service creation, nonfinancial outcomes, and financial outcomes. A key gap in the literature is the most studies have relied on product-based innovation studies to classify service innovation. We hope that study provides an impetus for more efforts in this area.
\end{abstract}

M. Rokonuzzaman $(\bowtie) \bullet$ A. Paswan

University of North Texas, Denton, TX, USA

e-mail: Md.Rokonuzzaman@unt.edu; Paswana@unt.edu 